Document downloaded from:

http://hdl.handle.net/10251/165401

This paper must be cited as:

Moreno, JM.; Velty, A.; Vidal Moya, JA.; Díaz Morales, UM.; Corma Canós, A. (2018).

Growing modulator agents for the synthesis of Al-MOF-type materials based on assembled 1D structural sub-domains. Dalton Transactions. 47(15):5492-5502.

https://doi.org/10.1039/C8DT00394G

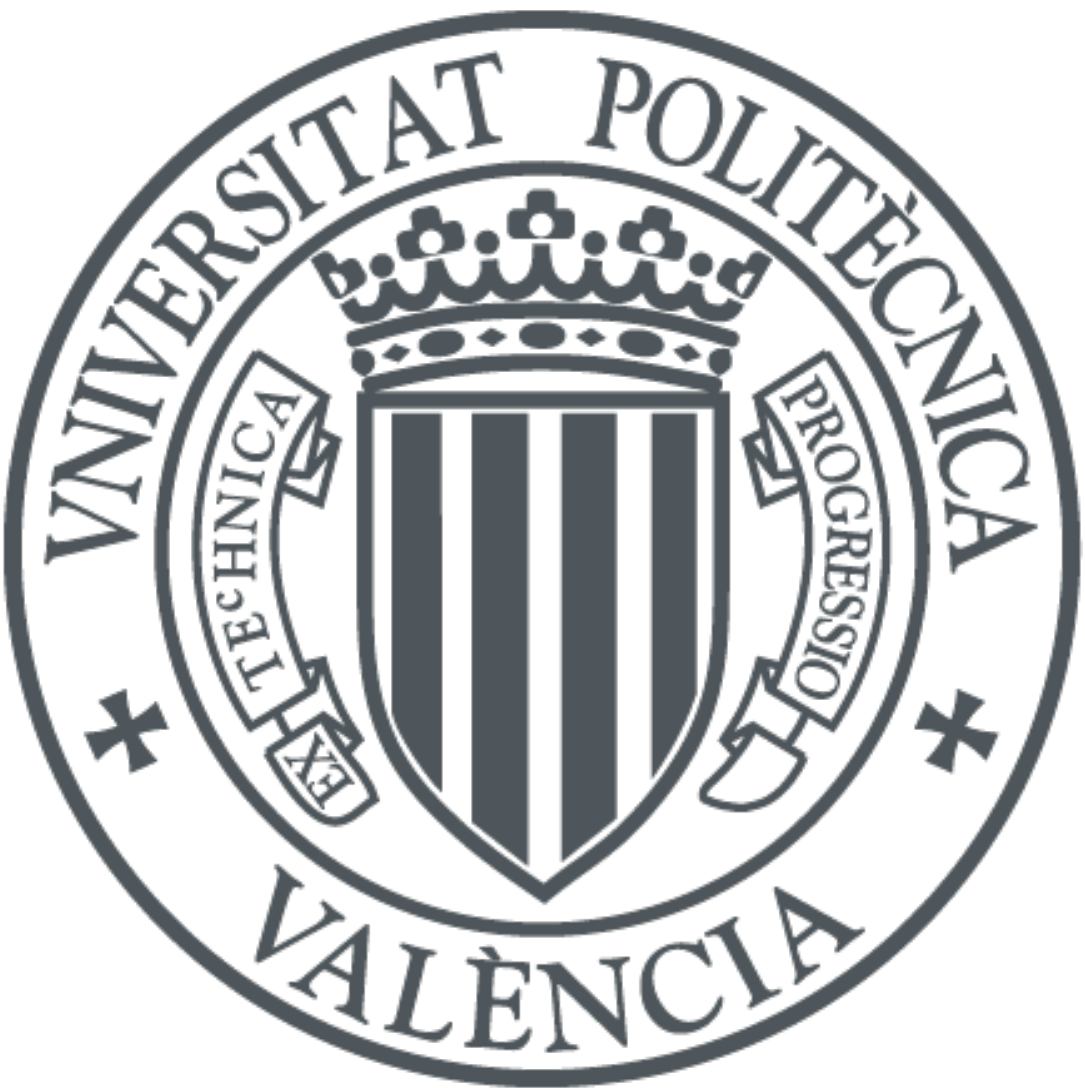

The final publication is available at

https://doi.org/10.1039/C8DT00394G

Copyright The Royal Society of Chemistry

Additional Information 


\title{
Growing modulator agents for the synthesis of Al-MOF-type materials based on assembled 1D structural sub-domains
}

\author{
José María Moreno, ${ }^{a}$ Alexandra Velty, ${ }^{a}$ José A. Vidal-Moya, ${ }^{a}$ Urbano Díaz*a and Avelino Corma*a \\ Novel aluminium MOF-type materials structured by 1D sub-domains like organic-inorganic nanoribbons are synthesized \\ modifying solvothermal synthesis conditions and solvents nature, in presence of mono-carboxylate aryl linkers with long \\ alkyl chains which act as growing modulator agents. Specifically, three different families of materials are prepared with \\ various morphological characteristics: (i) isoreticular MIL-53(Al)-type materials, (ii) mesoscopic metalorganic structures and \\ (iii) aluminium lamellar MOFs. The alkyl chain length in the aryl linker and the hydrophobic - hydrophilic nature of the \\ solvothermal synthesis media determine the structuration level achieved. The derived Al-MOFs are active and stable \\ catalysts for the synthesis of fine chemicals. This is illustrated by the efficient synthesis of 2,3-dihydro-2,2,4-trimethyl-1H- \\ 1,5-benzodiazepine.
}

\section{Introduction}

The use of suitable building blocks in optimal synthesis conditions has allowed the generation of new families of advanced porous materials with high gas uptake capacities, catalytic applications or technological functionalities (in sensoring, magnetism, optoelectronic or photoluminiscent fields). ${ }^{1-8}$ The active sites present in the individual builder units together with their intrinsic physico-chemical properties facilitate the preparation of a large number of organic-inorganic hybrid nanomaterials with a large variety of isolated functions which can modulate their behavior in function of the stable network in where they are included.

Usually, sol-gel, solvothermal and hydrothermal synthesis approaches have been useful to achieve an ideal auto-assembly of organic, inorganic and organic-inorganic building blocks to generate stabilized ordered hybrid materials with elevated accessibility and porous volume..$^{9,10}$ Specifically, bridged silsesquioxanes, aromatic derived linkers and metallic nodes have allowed the formation of periodic mesoporous organosilicas (PMOs), covalent organic frameworks (COFs) and metalorganic frameworks (MOFs), respectively, which are based on rigid networks through strong coordination or covalent connections established between the individual building blocks, being even prepared in absence of sophisticated structural directing agents (SDAs). ${ }^{11-13}$ However, although the number of porous hybrid materials is growing, they normally refer to 3D stabilized networks, being nowadays difficult to address the synthesis pathways towards the direct preparation of versatile and flexible 1D or 2D organic-inorganic solids. ${ }^{14}$

1D like-nanoribbons and 2D lamellar architectures are very interesting due to the associated structural versatility that allows the spatial modification of their individual nanostrips or nanolayers to generate more accessible materials or novel modified nanocomposites with additional functions incorporated onto the individual $1 D$ or 2D structural components..$^{15-17}$ In the case of hybrid materials, it is not usual to directly obtain, during the synthesis processes, lamellar organic-inorganic frameworks in only one-step mechanism. Specifically, 2D derived hybrid materials based on MWW or FER zeolitic layers can be obtained after multi-step post-synthesis processes in which the preparation of layered zeolitic precursors followed by consecutive swelling, pillarization, exfoliation or anchoring steps are required to homogeneously introduce additional organic or organometallic complexes onto the zeolitic surface. ${ }^{18-20}$

However, in the last years, important advances have been carried out in the preparation of 2D organozeolitic materials with the use of dual structural directing agents capable to form crystalline zeolitic nanosheets (MFI, BEA and MWW) by the interruption of the conventional $3 D$ individual crystals growing. ${ }^{17,21,22}$ Also, recently, lamellar pillared aluminosilicates with specific functional bridged silsesquioxanes have been prepared from rich aluminum alkaline synthesis gels under hydrothermal conditions in only one-step. ${ }^{23-25}$

In the case of hybrid materials based on metalorganic networks, the number of 1D or 2D organized structures is still more reduced than in organosiliceous solids. Few examples of poorly crystalline coordinated polymers structured from rare earth metallic nodes and highly hydrated have been described, together with pillared non-modifiable rigid MOFs obtained by the use of two aryl-dicarboxylate linkers perpendicularly located around metallic builders. ${ }^{26,27}$ Recently, deceleration of the solvothermal synthesis process has also been studied by means of different solvents mixtures, hindering the diffusion of reactive nutrients and, as result, stopping the growing of 3D MOFs facilitating the formation of small metalorganic nanosheets, though with reduced yields. ${ }^{28}$

The detection of clearly identifiable 2D lamellar and 1D structural sub-domains in 3D well-known MOFs, such as MIL-53, UiO-66, DUT-4, DUT-5, Cu(tpa) or MIL-68(Al), indicate that the synthesis processes of metalorganic frameworks could be based on the formation of low-dimensional sub-structures as starting 
precursors. ${ }^{29-35}$ Then, in this work, we propose the use of specific long-alkyl aromatic mono-carboxylate linkers, combined with metallic nodes, to favor the formation of 1D metalorganic ribbon-type structural sub-units which would be assembled to generate novel organized MOF-type solids under solvothermal conditions with different level of structuration and dimensionality. The combination of two solvents with different polarity during the synthesis process was decisive to obtain Al-MOFs with several morphologies and particular textural properties. Nevertheless, in all cases, 1D structural subdomains were present in the obtained metalorganic frameworks. The solids have been characterized by XRD, chemical analysis, TGA, NMR and IR spectroscopies, and FESEM/TEM microscopy to unreveal their physico-chemical characteristics. We will show that these hybrid materials are successful solid catalysts for the production of 2,3-dihydro2,2,4-trimethyl-1H-1,5-benzodiazepine (named as 1,5benzodiazipine). These compounds are of pharmaceutical interest as anticonvulsant, analgesic, sedative, anti-depressive, anti-inflammatory, and antibiotic agents. ${ }^{36-40}$ High yield of the products can be achieved under mild conditions.

\section{Results and discussion}

\section{Synthesis and Characterization}

The methodology followed to obtain three different families of Al-MOF-type materials formed by the coordinated autoassembly of organic and inorganic 1D builder sub-units was based not only on the use of specific organic spacers with just one carboxylate reactive point, but also modifying the solvothermal synthesis conditions, such as temperature, pressure, time or nature of the solvents. Specifically, monocarboxylate alkyl benzene compounds with hydrocarbon chains of different length, ethyl (EB), heptyl (HB) and dodecyl (DB), in all cases located in para position with respect to the carboxylic group, were used as molecular spacers. The XRD patterns obtained for each family of materials was different, confirming that the assembly of building blocks (monocarboxylate aromatic linkers and metallic clusters) occurred through different assembly routes as a function of synthesis methodology, solvent nature and type of alkyl-aryl linker used as spacer agent.

The first class of Al-metalorganic family was obtained by using only water as solvent in the synthesis medium (MIL53(Al)-type). Isoreticular hybrid frameworks were observed by XRD patterns being similar to conventional 3D MIL-53(Al), independently of the presence of long-alkyl aromatic linkers (EB and $\mathrm{HB}$ ) in the solvothermal process (Fig. 1-I). It is noteworthy that, in order to obtain Al-MOF materials with high crystallinity, it is necessary more prolonged synthesis times when longer alkyl chains are present in the linkers. This fact could be associated with the major difficulty of long hydrocarbon tails to be stabilized and spatially accommodated between the inorganic counterpart sub-units, or also due to higher entropy of the synthesis process. As consequence, the Al-MOF-type material obtained using the organic linker with longer alkyl tail (MIL-53(Al)-HB) exhibited the XRD pattern with shifted reflection bands compared with standard 3D MIL-53(AI), suggesting more marked structural alteration in the formed metalorganic framework (Fig. 1-Ic).
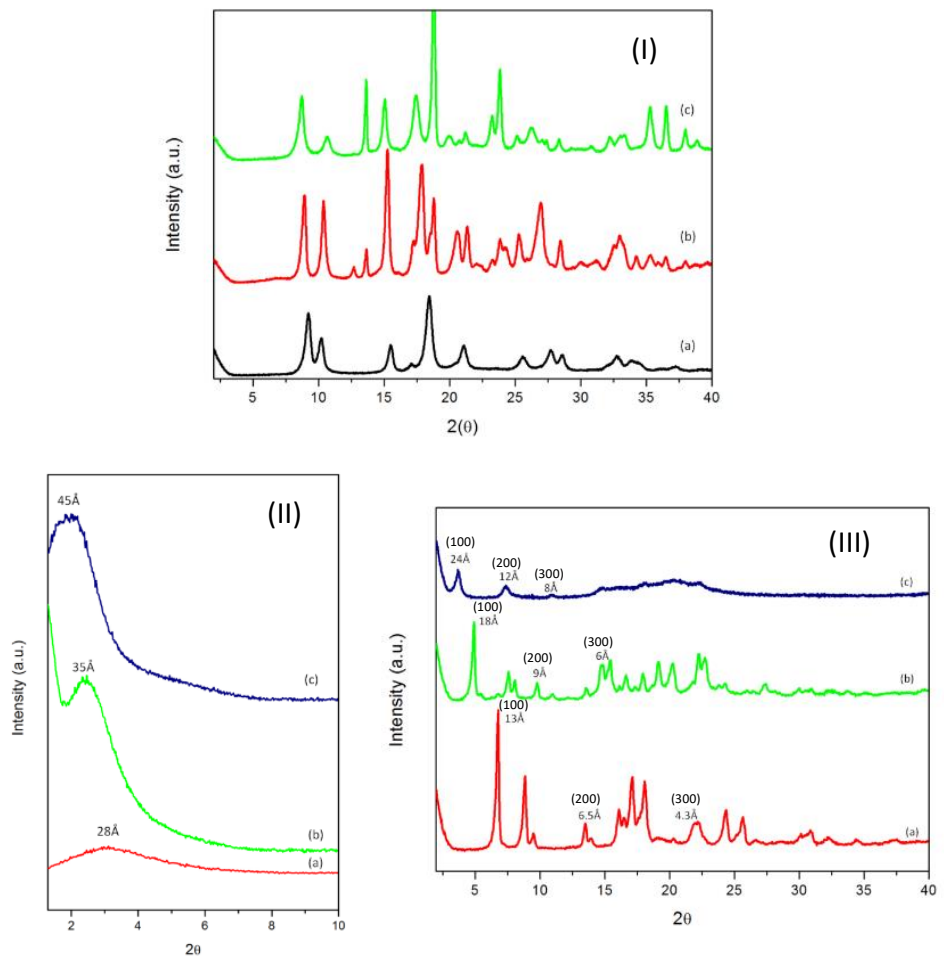

Fig. 1 (I) XRD patterns of isoreticular MIL-53(Al)-type MOFs: (a) 3D standard MIL-53(Al), (b) MIL-53(Al)-EB and (c) MIL-53(Al)-HB. (II) XRD patterns of mesoscopic samples: (a) Al-ITQ-EB, (b) Al-ITQ-HB and (c) Al-ITQ-DB. (III) XRD patterns of lamellar MOFs: (a) L-MOF-EB, (b) LMOF-HB and (c) L-MOF-DB.

On the other hand,

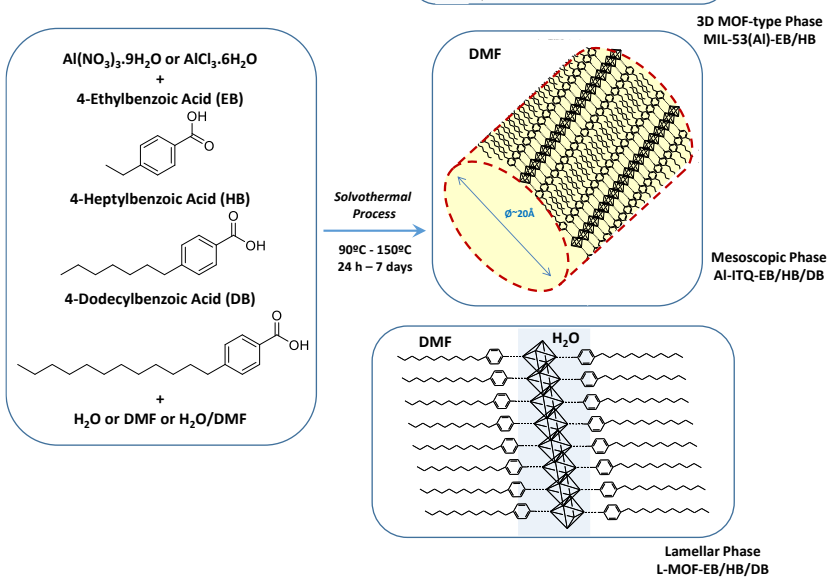

mesoscopic Al-MOF materials were synthesized when only DMF was used as solvent during solvothermal synthesis process. In this second family of Al-hybrid materials, XRD patterns showed one intense (100) diffraction band at low $2 \theta$ angles range at 28 $\AA$, $35 \AA$ and $45 \AA$ when $\mathrm{EB}, \mathrm{HB}$ and $\mathrm{DB}$ were used as organic spacers, respectively (Fig. 1-II). These characteristic bands were associated to mesoporous materials with long-range hexagonal ordering. As it is also observed in standard M41S-type materials, the formed mesoporous channels exhibited higher diameter when the length of the hydrocarbon chains was longer, being 
this fact confirmed by the position of (100) diffraction band at lower $2 \theta$ angles. Furthermore, the mesoscopic regularity achieved in these solids was poorer when the chain length of the organic spacer was shorter (EB), resulting in (100) diffraction bands with lower intensity.

Another kind of Al-MOF materials were synthesized when a mixture of solvents, DMF/Millia water, were used for the solvothermal synthesis. The XRD diffractograms showed that lamellar materials were prepared in presence of $E B, H B$ and $D B$ organic spacers, based on associated Al-metalorganic 1D nanoribbons separated by the long-alkyl monocarboxylate benzene groups, which were perpendicularly located to inorganic nodes. In fact, XRD patterns clearly show (100) low angle diffraction bands, which are characteristic of layered solids, formed by individual sheets perpendicularly disposed to axis $a$ (Fig. 1-III). Associated 1D organic-inorganic nanoribbons would be forming these sheets. Remarkably, the basal space obtained for the samples was different, indicating variation in the separation between the layers depending on the length of organic spacer used during the solvothermal process, which would act as effective growing modulators of the final lamellar metalorganic structures.

In this last family, the presence of (100) reflection band, together with the bands assigned to (200) and (300) diffraction orders (see (h00) indexing in Fig. 1-III), confirm that high regularity in the distance between the successive piled organicinorganic nanosheets has been achieved (Scheme 1), although the lamellar order is less homogeneous when the organic linker contains a longer alkyl tail (DB) (Fig. 1-IIlc). Furthermore, comparing with the XRD pattern of standard 3D Al-MIL-53 (Fig 1-la), the lamellar structures, L-MOF-EB, L-MOF-HB and L-MOF$\mathrm{DB}$, exhibited a completely different $\mathrm{X}$-ray diffractogram, being observable characteristic low angle (h00) bands together with the practical disappearance of standard (0kl) MIL-53(Al) diffraction bands, which would indicate the absence of $3 D$ framework's formation and the generation of novel 2D lamellar metalorganic architectures.

Specifically, (100) diffraction bands were located at $\sim 13 \AA$, $\sim 18 \AA$ and $\sim 24 \AA$ for derived MIL-53 materials synthesized in presence of organic modulators with ethyl-, heptyl and dodecylalkyl chains, respectively, being higher the separation between individual layers when longer linkers are used. Considering that the used organic spacers - EB, HB and DB - exhibited molecular lengths of $6.8 \AA$, $13.8 \AA$ and $20.0 \AA$, respectively, and that they were probably perpendicularly located in both sides of $1 \mathrm{D}$ inorganic $\mathrm{AlO}_{4}(\mathrm{OH})_{2}$ chains, present in the $1 \mathrm{D}$ nanoribbons, it would be possible to estimate that, in these three synthesized hybrid materials, the thickness of each individual 1D aluminiumchain was close to $\sim 4-5 \AA$ (Scheme 1). This result would imply the formation of lamellar hybrid materials based on ordered alternated 2D nanosheets based on associated $\left[\mathrm{AlO}_{6}\right]$ octahedral separated by long alkyl arylic spacers, though overlapping between organic chains of consecutive metalorganic layers may also occur. However, diffraction bands located around $10 \AA-11 \AA$, for L-MOF-EB and L-MOF-HB materials, would indicate that a certain level of structuration has been achieved in each individual metalorganic nanolayer.
Scheme 1 Synthesis routes to obtain aluminum-MOFs-type materials with different morphology, based on specific structural assembly of 1D nanoribbons, in function of solvothermal conditions.

The XRD results show that the solvothermal synthesis conditions and, specifically, the reaction media nature is decisive to modulate the morphology of the obtained hybrid materials. When only water is present in the synthesis slurry, the high organic content favors that $1 \mathrm{D}$ sub-domains are close together, generating an ordered and more compacted MOFtype structure similar to conventional 3D MIL-53(Al) architectures. An opposite effect is observed when only DMF is used as solvent during the synthesis. Because of the high hydrophobic affinity between organic linkers and the reaction medium, an elevated dispersion of structural sub-domains is facilitated, which are finally organized through mesoscopic morphology. Intermedium spatial phenomenon is detected in the organization of associated 1D nanoribbons units when $\mathrm{DMF} /$ water mixtures are present in the solvothermal process. In this latter case, organic tails, from specific spacer monomers, and aluminum octahedral strongly interact with DMF or water, respectively, achieving homogeneous and regular lamellar organization, due to the formation of successive organic and inorganic separated regions in the synthesis medium (Scheme 1). However, taking account the synthesis methodology used, the presence of small extra-framework alumina clusters as traces in the solids is not completely ruled out, although being practically undetectable from XRD patterns.

The structural hypotheses above commented were supported by the morphology observed for the three families of materials of derived Al-MOFs solids from electronic microscopy images (TEM and FESEM). Fig. 2a and Fig. S1 show TEM micrographs of the isoreticular MIL-53(AI) MOFs obtained in presence of monocarboxylate long-alkyl aromatic spacers using water as only solvent in the solvothermal synthesis process. In this case, it is observed the formation of crystals with size and topology similar to conventional 3D MIL-53(Al), although stratification is detected in the surface. This would be indicative of the presence of associated 1D sub-domains forming piled layers, assembled between them. These morphological characteristics were also observed from TEM and FESEM images (see ESI Fig. S1 and Fig. S2).
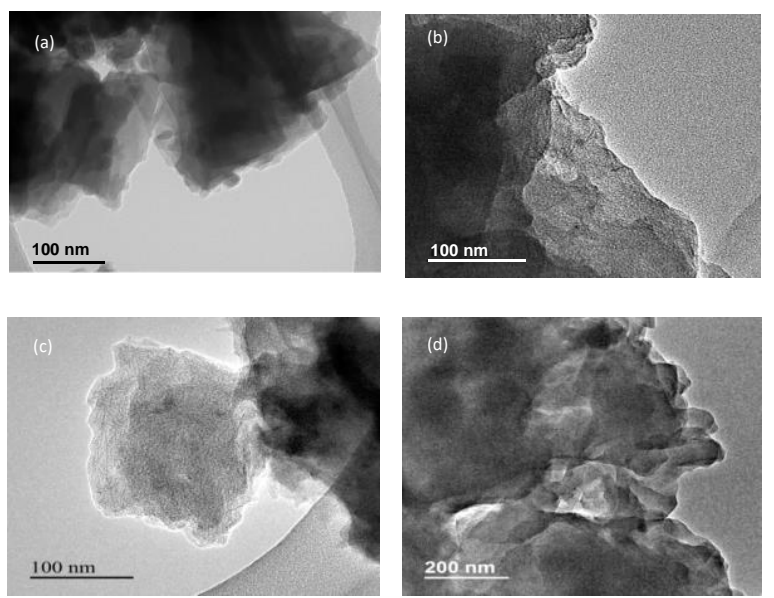
Fig. 2 TEM images: (a) MIL-53(Al)-HB, (b) and (c) Al-ITQ-HB, and (d) LMOF-HB samples. Scale bars correspond to $100 \mathrm{~nm}$ for (a), (b) and (c) micrographs, and $200 \mathrm{~nm}$ for (d) micrograph.

Furthermore, Fig.2b, Fig. 2c and Fig. S3 show TEM images of the samples synthesized only in presence of DMF as non-polar solvent. Micrographs show crystals with poor crystallinity and irregular morphology, indicating low homogeneity and nonordered structuration. In these samples, the mesoscopic longrange order was observed from TEM microscopy. However, the presence of free mesoporous was detected with difficulty because of unstable organic counterpart of the Al-MOFs materials under electronic beam. These mesoporous were distributed along plate-like crystals. The electronic microscopy images also showed the presence of areas formed by short sheets which would evidence the formation of lamellar substructures from associated 1D builder units. In this case, metalorganic nanolayers would probably be assembled around non-ordered mesocavities. However, the low structural periodicity exhibited by this type of hybrid solids along the channel direction would hinder the easy detection of the 1D sub-domains through electronic microscopy (see ESI Fig. S3).

TEM images of the hybrid materials obtained using DMF/MilliQ water mixtures as solvent (Fig. $2 d$ and Fig. S4) corroborate their lamellar nature. Overall, the sample containing heptyl tails as separator groups (L-MOF-HB) exhibits crystals formed by little plate-type crystallites, characteristic of layered materials, which are agglomerated between them, being even possible observing ordered individual layers inside each crystal (see ESI Fig. S4c). In the case of hybrid materials prepared with linkers containing shorter alkyl tails (L-MOF-EB), the lamellar effect is observed with difficulty probably due to the possible coexistence between lamellar and more compacted MIL-53-type sub-structures (see ESI Fig. S4).

The presence of structural sub-domains in the derived AlMOFs materials, because the existence of organic-inorganic 1D nanoribbons based on chains of associated aluminum octahedral nodes separated by pending monocarboxylate alkyl aromatic spacers, was confirmed through post-synthesis treatments of the as-synthesized solids with solvents which exhibited different polarity (methanol, dichloromethane (DCM), diethyl ether (DEE) or toluene). The solutions obtained were analysed by transmission electron microscopy (TEM) confirming that isolated short 1D nanoribbons, generated after the expansion and exfoliation of pristine metalorganic structures, formed the derived MOF-type materials. Nevertheless, the stability and durability of the colloidal suspensions was dependent of Al-MOF physico-chemical characteristics and solvent nature. Specifically, isoreticular MIL-53(AI) (see ESI Fig. S5) solids were only partially expanded in methanol and DEE in agreement with their more closed structure. On the contrary, more opened frameworks, such as those obtained in presence of DMF or DMF/MilliQ water mixtures (mesoscopic and lamellar metalorganic phases), were easily exfoliated in methanol, DEE or DCM (Fig. 3 and Fig. S5). Toluene was not suitable to expand the derived Al-MOFs materials, probably due to its high hydrophobic nature. The presence of homogeneously distributed hydrophobic and hydrophilic sub-domains in the metalorganic networks would explain the higher capacity of organic solvents with elevated polarity and dielectric constant to penetrate and exfoliate Al-nanoribbons from as-synthesized solids.
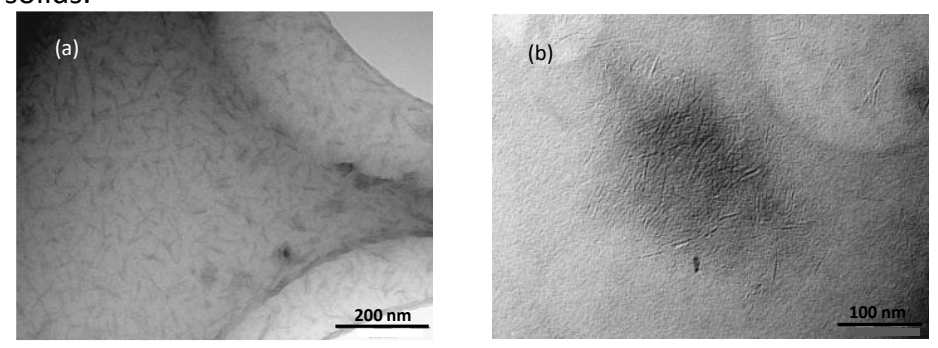

Fig. 3 TEM images: (a) Al-ITQ-HB and (b) Al-ITQ-DB samples exfoliated by dichloromethane solution. Scale bars correspond to $200 \mathrm{~nm}$ and $100 \mathrm{~nm}$ for (a) and (b) micrographs, respectively.

The chemical analysis of derived Al-MOF materials obtained at different synthesis conditions and using several organic spacers are shown in Table S1, Table S2 and Table S3. The high carbon content observed for all materials confirmed the effective incorporation of alkyl spacers in the metalorganic frameworks. In the case of isoreticular MIL-53(Al)-type family prepared in presence of only water as solvent, the organic content was close to that present in standard 3D MIL-53(Al) MOFs ( $40 \% \mathrm{wt})$, independently of the organic spacers nature used in the solvothermal process (see ESI Table S1). This fact should confirm that organic and inorganic counterparts are organized and spatially assembled to generate hybrid materials with similar topology to conventional 3D Al-MOFs, being this type of structuration favored by the presence of polar solvents (Scheme 1).

In the case of mesoscopic metalorganic materials obtained when DMF was used as only solvent, the organic content of the solids AI-ITQ-EB and Al-ITQ-HB was lower than in 3D MIL-53 (AI) MOFs ( 20-30\% wt). This fact is probably associated to the presence of a minor amount of organic spacers in the formed hybrid architectures. This can be due to a more difficult structuration and assembly between organic linkers and aluminum nodes, as consequence of high dispersion of reagents in the DMF synthesis medium that hinders their effective interconnection. In the case of derived mesoscopic Al-MOF containing dodecyl- units as organic counterpart (AI-ITQ-DB), the carbon content was higher due to the major molecular weight of the hydrocarbon tails present in the network (see ESI Table S2).

The highest organic content, $60 \% \mathrm{wt}$, was detected for AlMOF materials with

lamellar

structuration,

synthesized

in

DMF/MilliQ water

(L-MOF-EB, L-MOF$\mathrm{HB}$ and L-MOF-DB). This result would explain the high regularity achieved in the spatial separation of the associated 1D subdomains, formed by inorganic

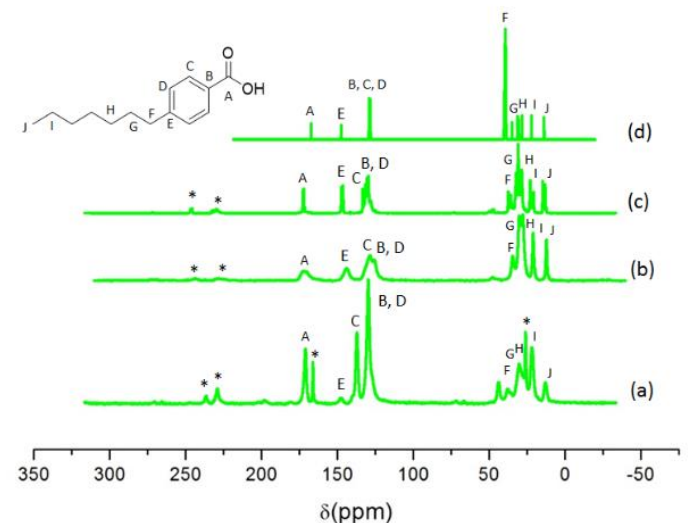


Al-octahedra and organic chains, due to the elevated number of homogenously distributed organic separators located in the interlayer space (see ESI Table S3).

It is important to point out that, when DMF was used during the synthesis process, nitrogen content was not observed in the solids, corroborating that the solvent molecules were completely removed during the washing and recovery of the derived Al-MOF materials.

From TGA curves and their corresponding DTA, organic amount present in the hybrid metalorganic solids and their thermal stability were determined (see ESI Fig. S6, Fig. S7 and Fig. S8). In all cases, without considering the contribution of hydration water and residual DMF molecules used as solvent in the solvothermal synthesis processes (both detected at around $80-150$ ㅇ range temperature), two main weight loses were clearly observed. The first, between $300^{\circ} \mathrm{C}$ and $400^{\circ} \mathrm{C}$, probably assigned to the presence of hydrocarbon tails from monocarboxylate para-alkyl benzene molecules used as spacers. In this weight loss, the contribution of water formed by dehydration of $\mathrm{AlO}_{4}(\mathrm{OH})_{2}$ builder units present in the 1D inorganic nanoribbons could also be included. Precisely, this phenomenon associated with the oxygen elimination present in the $\left[\mathrm{AlO}_{6}\right]$ octahedral groups would explain the higher organic content detected from TGA compared with CHNS analysis (see ESI Table S1, Table S2 and Table S3). The second weight loss, observed within the $500-600 \circ \mathrm{C}$ range temperature, was assigned to aromatic rings from organic spacers coordinated to inorganic Al-octahedra, which are present in all derived AlMOFs materials.

The stability of the samples was also analysed by XRD obtained after successive treatments at increasing temperatures. The diffractograms showed that the isoreticular MIL-53(Al) and lamellar phases are stable up to $200 \circ \mathrm{C}-300 \circ \mathrm{C}$, being generated the same collapsed structure based on aluminium oxi-hydroxides when the organic spacers were progressively removed (see ESI Fig. S9 and Fig. S10). The stability of the mesoscopic phase was lower and the (100) diffraction band, characteristic of long-range hexagonal order, disappears when the samples were treated at temperatures close to $200 \circ \mathrm{C}$

\section{Spectroscopic characterization}

${ }^{13} \mathrm{C}$ CP/MAS NMR spectra of MIL-53(Al)-HB, Al-ITQ-HB and LMOF-HB samples are shown in Fig. 4. In all cases, the total integrity of the organic spacers (HB) after the solvothermal synthesis processes was confirmed since all carbon atoms, including the hydrocarbon tails directly bonded to monocarboxylate benzene units, were univocally assigned in the ${ }^{13} \mathrm{C}$ NMR spectra (see the inset in Fig. 4 together with ${ }^{13} \mathrm{C}$ NMR spectrum of pure organic $\mathrm{HB}$ compound). Specifically, chemical shifts between 120 and 160 ppm were observed which are assigned to carbon atoms in aromatic rings, while the band at $\sim 170 \mathrm{ppm}$ is associated to arylcarboxylate groups. Furthermore, different bands were detected between 10 and 50 ppm chemical shifts assigned to carbon atoms $-\mathrm{CH}_{2}-$ from alkyl tails of the $\mathrm{HB}$ organic spacers. Thus, these results corroborated that the monocarboxylate alkyl benzene groups, used during the synthesis process, and coordinatively attached to octahedral aluminum nodes, remained intact as in the initial chemical conformation, being finally included in the derived $\mathrm{Al}$ MOF solids.

Fig. $4{ }^{13} \mathrm{C}$ MAS NMR spectra: (a) MIL-53(Al)-HB, (b) Al-ITQ-HB, (c) LMOF-HB and (d) pure 4-heptylbenbzoic acid. Signals with asterisk correspond to spinning bands.

Additionally, chemical aluminium environment was determined by ${ }^{27} \mathrm{Al}$ MAS NMR spectroscopy (Fig. 5), being confirmed that practically all aluminium is located in octahedral positions integrating the inorganic chains present in the 1D nanoribbon-type structural sub-units. Al-ITQ-HB sample, exhibited a well-defined peak centered at $\sim 6.5 \mathrm{ppm}$ chemical shift that is characteristic of aluminium (oxo)hydroxide species (Fig. 5b), showing a regular octahedral environment of metallic atoms conforming the inorganic counterpart of highly homogeneous mesoscopic structuration. It is noteworthy that aluminium overlapped signals were detected with a quadrupolar character from the spectra of the Al-MOF solids synthesized in presence of water (MIL-53(Al)-HB) and DMF/MilliQ water mixtures (L-MOF-HB) (Fig. 5a and Fig. 5c). This effect is characteristic of solvated octahedral aluminium interacting with water molecules, present in the solvothermal reaction medium, that favour broad chemical shift signals because there is now an electric field gradient at the aluminium structural nodes. ${ }^{41}$

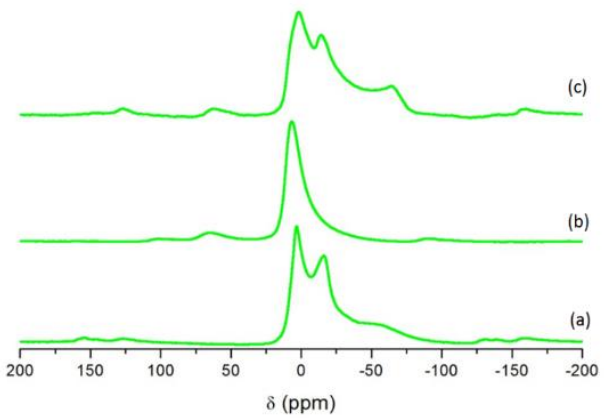

Fig. $5{ }^{27} \mathrm{Al}$ MAS NMR spectra: (a) MIL-53(Al)-HB, (b) Al-ITQ-HB and (c) L-MOF-HB.

On the other hand, 2D ${ }^{1} \mathrm{H}$ to ${ }^{13} \mathrm{C}$ and ${ }^{1} \mathrm{H}$ to ${ }^{27} \mathrm{Al}$ (HETCOR) $\mathrm{CP}$ MAS NMR spectra indicated the close proximity between octahedral aluminum nodes with carboxylate aromatic rings and aliphatic carbon atoms (Fig. 6). In detail, 2D spectra of mesoscopic Al-ITQ-HB material showed a cross correlation between aromatic and aliphatic protons of 4-heptylbenzoate groups at $7 \mathrm{ppm}$ and $1.5 \mathrm{ppm}$, respectively, with the ${ }^{27} \mathrm{Al}$ signal

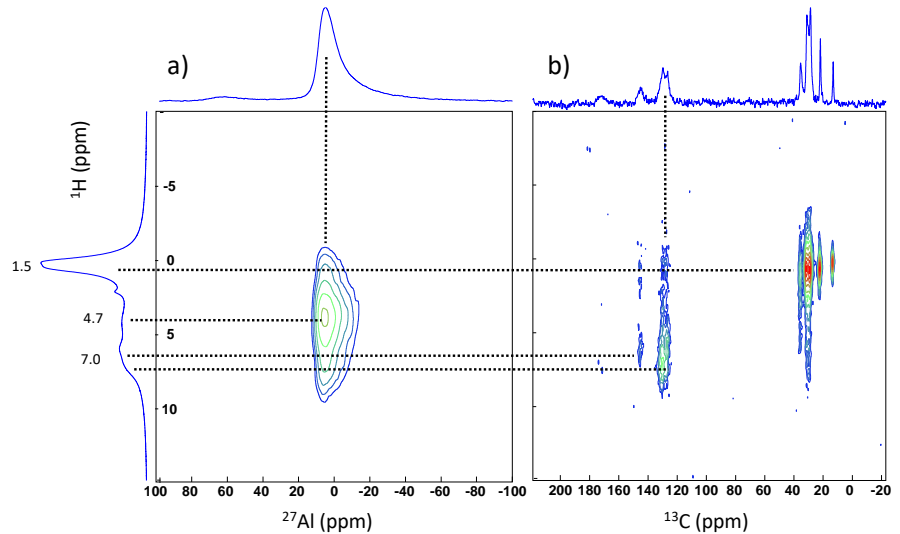


at $6.5 \mathrm{ppm}$. This result would suggest the effective formation of organic-inorganic 1D nanoribbons as structural sub-units, which would be associated between them to form derived Al-MOFtype materials with several topologies in function of solvothermal synthesis conditions.

Fig. $62 \mathrm{D}$ (a) ${ }^{1} \mathrm{H}$ to ${ }^{27} \mathrm{Al}$ and (b) ${ }^{1 \mathrm{H}}$ to ${ }^{13} \mathrm{C}$ (HETCOR) CP-MAS NMR spectra of mesoscopic AI-ITQ-HB sample.

Furthermore, the integrity of organic spacers units included in the framework of hybrid materials was also confirmed by IR spectroscopy (see ESI Fig. S11, Fig. S12 and Fig. S13). The presence of aliphatic (C-H) chains from alkyl long tails of the organic spacers (ethyl, heptyl and dodecyl) was corroborated by different vibration signals around $3000 \mathrm{~cm}^{-1}$, being identified three bands at 2983, 2953, $2885 \mathrm{~cm}^{-1}$ associated to $-\mathrm{CH}_{2-}$ groups. The IR results also showed the presence of stretching vibration mode at $3600-3200 \mathrm{~cm}^{-1}$ that corresponds to the hydroxyl groups $(-\mathrm{OH})$, associated to hydration water and aluminum oxo-hydroxide structural units $\left(\mathrm{AlO}_{4}(\mathrm{OH})_{2}\right)$. Moreover, a typical vibrational area due to the carbonyl group of the organic ligands bond to Al octahedral units is observed at $1400-1600 \mathrm{~cm}^{-1}$. Further signals located between 1400 and $1600 \mathrm{~cm}^{-1}$ were assigned to asymmetric stretching vibrations due to the presence of ring aromatic and carboxylate groups included in the organic-inorganic network. So, IR spectroscopy verified unambiguously the presence and integrity of the organic spacers forming the hybrid structure, also confirming the interpretation of the previously showed NMR results.

On the other hand, Lewis acidity of the derived metalorganic materials was analysed from FTIR spectroscopy by CO adsorption. Specifically, CO adsorption at low temperatures $(100 \mathrm{~K})$ was controlled by FTIR technique to obtain information about Lewis acid sites present in Al-MOF-type solids (see ESI Fig. S14, Fig. S15, Fig. S16 and Fig. S17). In all cases, an absorption band is observed at approximately $2150 \mathrm{~cm}^{-1}$, indicating that weak Lewis acid sites are included in the hybrid frameworks. This fact was corroborated by the complete disappearance of this band after subsequent treatment of the samples at $100 \mathrm{~K}$ in vacuum.

\section{Textural properties}

Textural properties of derived Al-MOF materials prepared were analysed from Argon adsorption isotherms (see ESI Fig. S18) for materials synthesized in presence of heptylbenzoic acid as organic spacer. BET specific surface areas were different depending on the solvent used for the synthesis of the metalorganic materials (water, DMF or DMF/Millia water mixtures). In detail, isoreticular MIL-53(Al)-HB material exhibited type I isotherm, characteristic of microporous materials, achieving the highest specific surface area $\left(\mathrm{S}_{\mathrm{BET}}=383\right.$ $\mathrm{m}^{2} / \mathrm{g}$ ) associated to similar MIL-53(Al)-type structural organization (Scheme 1). In the case of mesoscopic phases (AlITQ-HB), specific surface area was lower $\left(\mathrm{S}_{\mathrm{BET}}=30 \mathrm{~m}^{2} / \mathrm{g}\right)$ due to the poor crystallinity and short order structuration achieved. Furthermore, the elevated concentration of organic spacers blocking porous volume and their elevated hydrophobic nature could hinder the optimal gas adsorption between the 1D organic-inorganic Al-nanoribbons. This problem would also be present in the lamellar hybrid materials such as L-MOF-HB solid $\left(\mathrm{S}_{\mathrm{BET}}=70 \mathrm{~m}^{2} / \mathrm{g}\right)$.
Hörvath-Kawazoe pore size distribution showed that the majority of pores were centered at approximately $6 \AA$ for isoreticular MIL-53(Al)-HB material due to associated microporosity characteristic of more compacted networks. However, for mesoscopic AI-ITQ-HB and lamellar L-MOF-HB materials the pore size distribution was centered at approximately 20-25 $\AA$, being this value indicative of the presence of mesoporous cavities probably following hexagonal array or due to the galleries formed between inorganic aluminum chains and long alkylic tails located in the interlayer space (Scheme 1 and Fig. S19).

\section{Catalytic Activity}

Possible application of this new family of derived Al-MOFs, based on 1D organic-inorganic nanoribbons, as acid catalyst was explored. The use of MOFs with coordinatively unsaturated sites (CUS) has been widely studied. When one of the coordination positions of the metal centers is occupied by a weakly coordinated ligand, this can be easily removed without collapse of the crystalline structure. Thus, the presence of CUS in the polyhedral inorganic nodes confers the Lewis acidity to MOFs. The ability to vary both textural and structural properties, using monocarboxylate benzylic ligands, allows variation in the amount and dispersion of CUS in the framework. ${ }^{42}$

From the catalytic point of view, we believe that MOFs should be used for the production of fine chemicals and, in general, for the preparation of molecules with a higher added value. ${ }^{43}$ Then, taking into account the large pore and flexibility of the materials presented here, we have studied their possibilities as catalysts for the production of benzodiazepines. Diazepines and benzodiazepines are widely used in pharmacy and have various therapeutic applications, finding uses as anticonvulsants, antianxiolitics, analgesics, sedatives, antidepressives and hypnotic agents. Also, benzodiazepine derivatives are used as dyes for acrylic fibers and are valuable intermediates in the synthesis of fused ring substrate such as triazolo-, oxadiazolo-, oxazino-, and furanobenzodiazepines. Generally, benzodiazepines are synthesized by condensation between o-phenylenediamines and carbonyl compounds in presence of both Lewis and Brönsted acids. For examples, polyphosphoric acid on $\mathrm{SiO}_{2},{ }^{44}$ acetic acid under microwave irradiation, ${ }^{45}$ nitrobenzoic acid, ${ }^{46} \mathrm{H}_{2} \mathrm{SO}_{4} / \mathrm{ZrO}_{2},{ }^{47}$ were used as Brönsted type catalysts to produce 1,5-benzodiazepine. In addition, HY, H-ZSM-548 and H-MCM- $22^{49}$ zeolites or mesoporous aluminosilicate (MCM-41) $)^{50}$ have been used as Brönsted-type acid catalysts. On other hand, different Lewis acids have been reported as efficient catalysts for the synthesis of benzodiazepines such as $\mathrm{ZnCl}_{2},{ }^{51} \mathrm{YbCl}_{3}, 52$ or $\mathrm{FeCl}_{3}$ supported over poly(4-vinylpyridine) polymer ${ }^{53}$ or other micro and mesoporous Fe-containing materials. ${ }^{54}$ In this paper, we studied the catalytic performance of the prepared hybrid materials, MIL53(Al)-HB, Al-ITQ-HB and L-MOF-HB, as well as the effect of their different morphology for the cyclocondensation of ophenylenediamine $(0.5 \mathrm{mmol})$ with acetone $(10 \mathrm{mmol})$ to provide 2,3-dihydro-2,2,4-trimethyl-1H-1,5-benzodiazepine (1, 5 benzodiazepine), at 65 으.

When the reaction was performed in the presence of MIL53(Al)-HB, the rapid formation of an imine was firstly observed as reaction intermediate, i. e. $\mathrm{N}^{1}$-(1-methylethylidene)-1,2benzenediamine (imine) (Scheme S1), then a low reaction of the 
imine with the acetone gives a diimine, which after the 1,3 shift of the hydrogen atom provides an imine-enamine, which easily cycles to form 1,5-benzodiazepines. ${ }^{55}$ Proposed mechanism of cyclocondensation over CUS as Lewis acid active sites present in the framework of Al-MOF-type materials is shown in Scheme 2. Also, 2-methyl-1,4-dihydroquinoxaline can be identified as subproduct, probably due to the direct imine intermediate cyclization. When the kinetics curves plotted in Fig. 7 were compared, a similar catalytic behavior was noted for both MIL53(Al)-HB and Al-ITQ-HB materials, while in the presence of LMOF-HB the initial rate of reaction, as well as the final yield of 1,5 benzodiazepine after 10 hours of reaction time were lower. Indeed, the 1,5 benzodiazepine yield were of 90,88 and $68 \%$ for MIL-53(Al)-HB, Al-ITQ-HB and L-MOF-HB, respectively; while a small amount of imine remained in the reaction medium in all cases, 4,8 and $16 \%$ for MIL-53(AI)-HB, Al-ITQ-HB and L-MOF-HB, respectively. The lower rate of reaction using L-MOF-HB catalyst was attributed to the higher organic content located in the interlayer space that difficult the diffusion into the internal porous system. The XRD study showed that mesoscopic AI-ITQHB catalyst was not stable after successive reaction cycles, under the performed reaction conditions (see ESI Fig. S20).

Likewise, when the reaction was carried out in the presence of 3D standard MIL-53(Al), whose structure contains a shorter organic ligand (terephthalic acid), high catalytic performance was observed for 1, 5-benzodiazepine production (Table 1 ), this result being comparable to that exhibited by MIL-53(AI)-HB due to similar compacted structure of both materials. These results showed the importance of the hydrophobic/hydrophilic properties of the catalytic surface as well as the morphology and accessibility to active sites. ${ }^{56}$ Furthermore, the high reactivity showed by MIL-53(AI)-HB was validated since conversion and selectivity achieved for 1, 5-benzodiazepine formation was comparable with that obtained with highly reactive conventional aluminum microporous zeolites or M41Stype mesoporous materials tested in similar reaction conditions. ${ }^{57}$

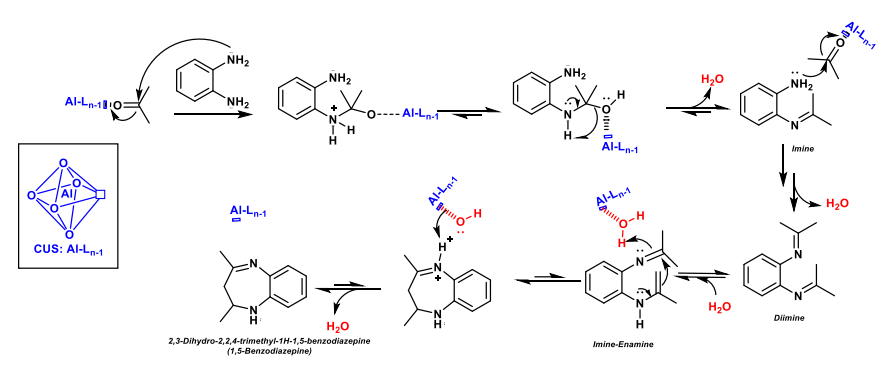

Scheme 2 Proposed mechanism of cylocondensation over CUS as Lewis acid active sites.

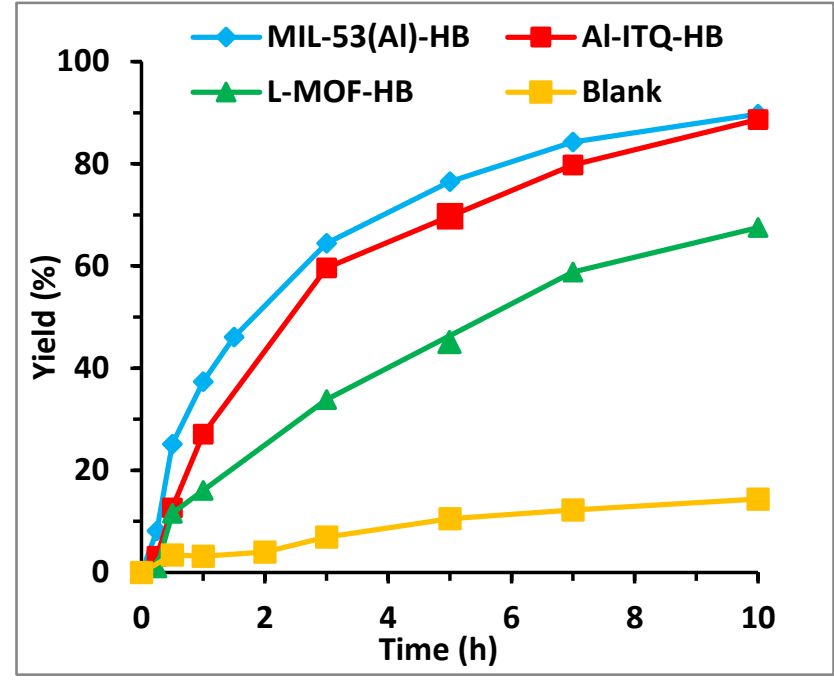

Fig. 7 Kinetics for 1,5-benzodiazepine production using MIL-53(Al)-HB, Al-ITQ-HB and L-MOF-HB as solid catalysts. Blank reaction is included.

Table 1 Yields and selectivity for 1,5-benzodiazepine productiona.

\begin{tabular}{|c|c|c|c|c|c|}
\hline \multirow{2}{*}{ Catalysts } & \multicolumn{2}{|c|}{ 1,5-benzodiazepine(\%) } & \multicolumn{2}{|c|}{ Yield (\%) } & \multirow{2}{*}{ 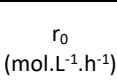 } \\
\hline & Yield & Selectivity & Imine & Subproduct $^{b}$ & \\
\hline MIL-53(Al)-HB & 90 & 90 & 4 & 6 & 0.30 \\
\hline Al-ITQ-HB & 88 & 88 & 8 & 4 & 0.19 \\
\hline L-MOF-HB & 68 & 68 & 16 & 16 & - \\
\hline MIL-53(AI) & 87 & 90 & 8 & 1 & 0.23 \\
\hline $\mathrm{HB}^{\mathrm{c}}$ & 45 & 50 & 37 & 8 & - \\
\hline Blank & 14 & 16 & 56 & 15 & - \\
\hline $\mathrm{v}-\mathrm{Al}_{2} \mathrm{O}_{3}$ & 2 & 2 & 73 & 13 & - \\
\hline
\end{tabular}

a Reaction conditions: Diamine $(0.5 \mathrm{mmol})$, acetone $(10 \mathrm{mmol}), 14 \mathrm{~mol} \% \mathrm{Al}$, at $65^{\circ} \mathrm{C}, 10 \mathrm{~h}$ b 2-Methyl-1,4-dihydroquinoxaline. c 4 -Heptylbenzoic Acid (5 mol\%).

Additionally, a study of leaching was performed. After 1.5 hour of reaction time, the MIL-53(AI)-HB catalyst was removed by filtration and the process was continued. From Fig. 8, a slight increase of the yield from 44 to $57 \%$ was observed, which can be due to the blank contribution (Table 1). An additional experiment with a 5 mol\% of p-heptylbenzoic acid (HB) was carried out to check the catalytic performance of the carboxylic acid as homogenous Brönsted acid. After 10 hours of reaction time, the maximum yield of 1,5 benzodiazepine was $45 \%$. Furthermore, catalytic activity of $\mathrm{y}-\mathrm{Al}_{2} \mathrm{O}_{3}$ in this reaction was estimated, being observed that the yield was practically zero. This fact evidenced the null contribution of small alumina traces, maybe present in the Al-MOF-type materials, in their final reactivity. With these results, we can conclude that no leaching was observed and that the high catalytic performance of MIL-53(Al)-HB to the preparation of 1,5 benzodiazepines can be exclusively assigned to the coordinatively unsaturated aluminum sites.

Finally, a study of stability and recyclability of MIL-53(AI)-HB was performed for four consecutive runs. From Fig. 9, we observed that the isoreticular MOF maintained its catalytic performance over the reuses, since only a slight decrease of 1,5 benzodiazepine yield was observed. While XRD patterns and 
NMR ${ }^{13} \mathrm{C}$ spectra showed the stability of the isoreticular framework throughout reuses and under studied reaction conditions (see ESI Fig. S21 and Fig. S22).

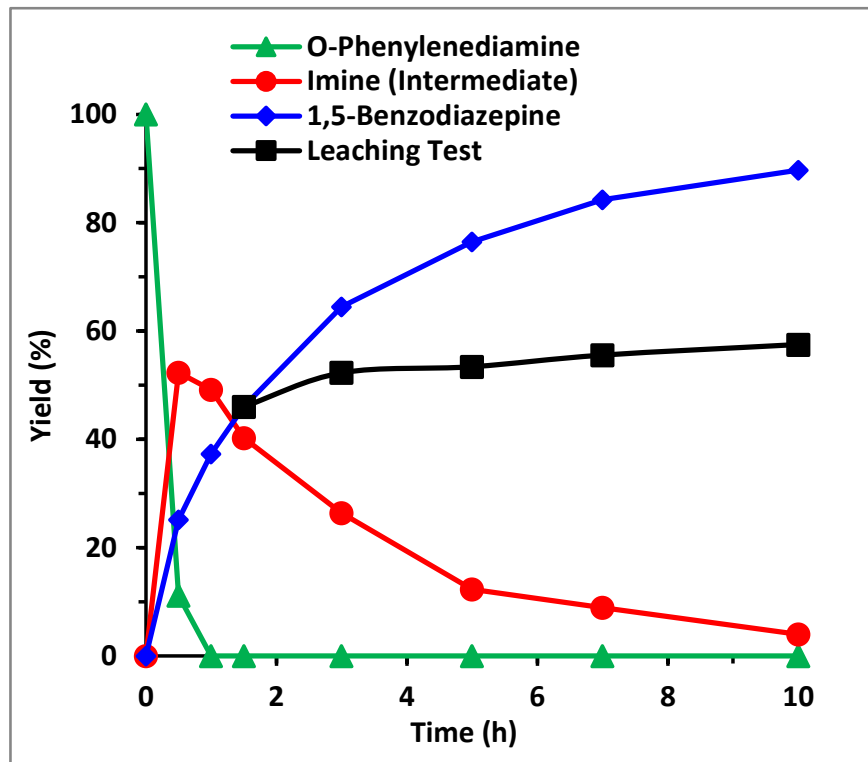

Fig. 8 Kinetics for 1,5-benzodiazepines production using isoreticular MIL-53(Al)-HB as catalyst. Yields of O-phenylenediamine, imine (intermediate), 1,5-benzodiazepine and leaching test are shown.

Definitively, these results allow us to conclude that isoreticular MIL-53(Al)-HB materials because of the presence of coordinatively unsaturated aluminum sites together with their hydrophobic/hydrophilic nature, are active and stable hybrid organic-inorganic acid catalysts to the synthesis of 1,5benzodiazepine. Similar tendency was also observed when EB organic spacers were present in the metalorganic networks to form isoreticular MIL-53(AI)-type structures (see ESI Table S4).

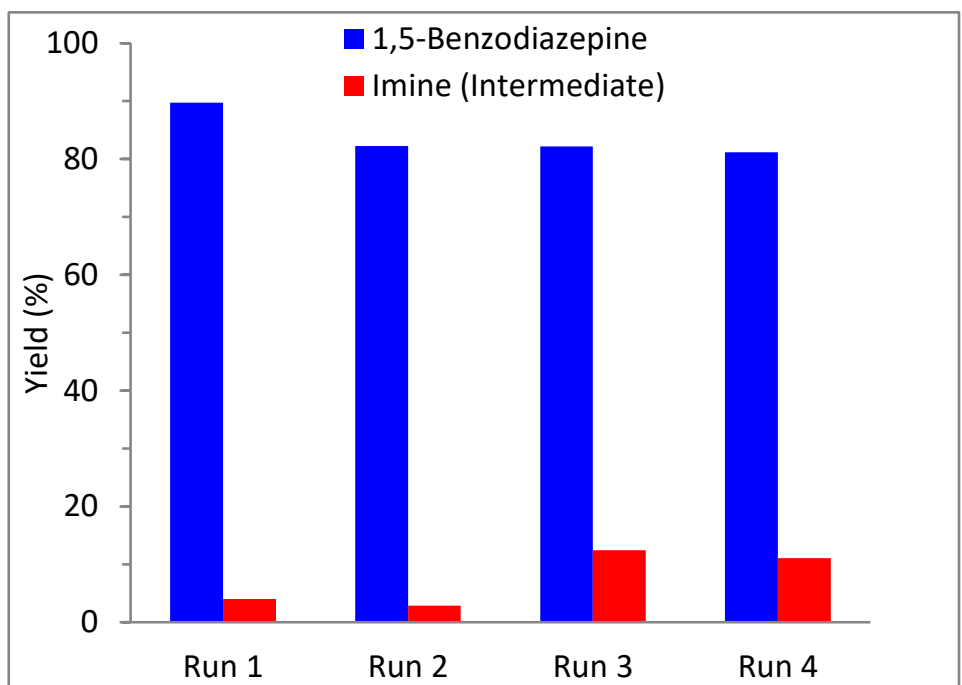

Fig. 9 Four uses for 1,5-benzodiazepine production using isoreticular MIL-53(Al)-HB as catalyst. Yields of first and second uses (10 h), third use $(13 \mathrm{~h})$ and fourth use $(20 \mathrm{~h})$ are shown.

\section{Conclusions}

We have successfully prepared novel families of hybrid metalorganic materials based on the effective assembly of 1D organic-inorganic ribbon-type structural sub-units at nanometric scale by the use of specific monocarboxylate longalkyl aromatic linkers, which are combined with aluminum nodes, under solvothermal conditions. The suitable combination of two solvents, during the synthesis process, with different polar nature was decisive to obtain these novel types of Al-MOFs with several morphologies and stabilities. Specifically, the use of water, DMF of DMF/water mixtures as synthesis reaction media are decisive to obtain isoreticular more compacted MOF-type structures, mesoscopic phases or lamellar organized hybrid architectures, respectively. However, in all cases, the same 1D structural sub-domains were identified in the as-synthesized derived aluminum-metalorganic frameworks through different characterization results and exfoliation post-synthesis treatments. The potentiality of these hybrid materials, as acid catalysts, was confirmed for the selective production of 1,5-benzodiazipines with interest in fine chemistry related with pharmacological industry.

\section{Experimental}

\section{Chemicals}

New families of aluminium-MOFs type materials were obtained using 4-ethylbenzoic acid (EB, Aldrich), 4-heptylbenzoic acid (HB, Alfa Aesar) and 4-dodecylbenzoic acid (DB, Alfa Aesar) as organic sources. Aluminium nitrate nonahydrate $\left(\mathrm{Al}\left(\mathrm{NO}_{3}\right)_{3} .9 \mathrm{H}_{2} \mathrm{O}\right.$, Aldrich) and aluminium chloride hexahydrate ( $\mathrm{AlCl}_{3} .6 \mathrm{H}_{2} \mathrm{O}$, Aldrich) were used as inorganic sources. Dimethylformamide (DMF, Across), MilliQ water or mixtures $\mathrm{DMF} /$ MilliQ water were used as solvents in solvothermal synthesis media. Methanol was used to remove of unreacted ligands and to exchange the solvent molecules retained during the solvothermal synthesis. 


\section{Synthesis of derived metalorganic materials}

Conventional 3D MIL-53(Al) was synthesized using 1,4 bencenedicarboxylic acid as organic linker (BDC, Aldrich) following the bibliography. ${ }^{29}$ Three different families of metalorganic materials were obtained by changing temperature, time and solvents during the solvothermal synthesis process.

\section{MIL-53(Al)-EB and MIL-53(Al)-HB}

MIL-53(AI)-EB and MIL-53(Al)-HB were obtained by separately mixing organic linker ( $E B$ or $H B$ ) and aluminium source $\left(\mathrm{Al}\left(\mathrm{NO}_{3}\right)_{3} .9 \mathrm{H}_{2} \mathrm{O}\right)$ in MilliQ water at room temperature [1Al:0.5linker: $8 \mathrm{H}_{2} \mathrm{O}$ ]. After, aluminium solution was added onto organic solution. The formed slurry was introduced into a stainless steel autoclave and heated at $150 \circ \mathrm{C}$ for 3 days (EB) or 7 days $(\mathrm{HB})$ under autogenous pressure and static conditions. Once cooled to room temperature, the solids were filtrated with distilled water and washed with methanol several times, being dried overnight at $60 \circ \mathrm{C}$ under vacuum.

\section{Al-ITQ-EB, Al-ITQ-HB and Al-ITQ-DB}

The synthesis methodology of this family of materials was carried out by mixing organic linkers (EB, $\mathrm{HB}$, or $\mathrm{DB}, 3.1 \mathrm{mmol}$ ) and aluminum reagent $\left(\mathrm{AlCl}_{3} .6 \mathrm{H}_{2} \mathrm{O}, 3.1 \mathrm{mmol}\right)$ in $15 \mathrm{~mL}$ of $\mathrm{DMF}$, each one separately. When both solutions were totally homogeneous, they were mixed together. Then, the formed solution was charged into a stainless autoclave and heated at $150^{\circ} \mathrm{C}$ for $24 \mathrm{~h}$ under autogeneous pressure and static conditions. After this, the autoclave was cooled to room temperature and the obtained solid was isolated by filtration with distilled water and washed several times with methanol, being dried at room temperature.

\section{L-MOF-EB, L-MOF-HB and L-MOF-DB}

The last family of materials was accomplished when the organic linker (EB, HB or $\mathrm{DB}, 5.33 \mathrm{mmol}$ ) was introduced into a flask bottle together with a mixture of MilliQ water/DMF ( $25 \mathrm{~mL}$ and $20 \mathrm{~mL}$, respectively) and heated at $90 \circ \mathrm{C}$ until the solution was clear. Then, the aluminum reagent $\left(\mathrm{Al}\left(\mathrm{NO}_{3}\right)_{3} .9 \mathrm{H}_{2} \mathrm{O}, 5.33 \mathrm{mmol}\right)$ was separately dissolved in water $(5 \mathrm{~mL})$ at room temperature, being added to the first solution at $90 \circ \mathrm{C}$. The gel was keep at $90 \circ \mathrm{C}$ for 5 days and the formed solid was recuperated by filtration with DMF and distilled water. Then, the recovered solid was washed several times with methanol and dried overnight at $60 \circ \mathrm{C}$ under vacuum.

\section{Catalytic activity: 1,5-benzodiazepine formation}

Condensation reactions were carried out in a closed conic vessel with magnetic stirring. A mixture of o-phenylenediamine $(0.5$ $\mathrm{mmol})$ and acetone $(10.0 \mathrm{mmol})$ at $65 \stackrel{\circ}{\circ}$ was magnetically stirred, and the necessary amount of solid catalyst to contain $14.0 \mathrm{~mol} \%$ of Al was added.

In all the cases, samples were taken periodically during the reaction and analyzed by GC/MS and GC equipped with an HP5 column $(30 \mathrm{~m} \times 0.32 \times 0.25 \mu \mathrm{m})$ and a FID as detector.

\section{Characterization techniques}

XRD analysis was carried out with a Philips X'PERT diffractometer equipped with a proportional detector and a secondary graphite monochromator. Data were collected stepwise over the $2 \circ \leq 2 \theta \leq 20$ angular region, with steps of 0.020 $2 \theta, 20$ s/step accumulation time and $\operatorname{CuK} \alpha(\lambda=1.54178 \AA)$ radiation. Transmission electron microscopy (TEM) micrographs were obtained with a JEOL JEM2100F electron microscope operating at $200 \mathrm{keV}$. The samples were prepared directly by dispersing the powders onto carbon copper grids. Fieldemission scanning electron microscopy (FESEM) micrographs were recorded on a ZEISS Ultra 55 microscope operating at $2 \mathrm{kV}$, with a $2 \times 10^{-9}$. C, N and $\mathrm{H}$ contents were determined with a Carlo Erba 1106 elemental analyser, while Al contents were obtained by means of atomic absorption spectroscopy (Spectra AA 10 Plus, Varian). Thermogravimetric and differential thermal analyses (TGA-DTA) were conducted in an air stream with a Metler Toledo TGA/SDTA 851E analyser. Ar adsorption isotherm was performed at -186 ㅇ $\mathrm{C}$ in an ASAP 2010 apparatus from Micromeritics, after pretreating the samples under vacuum at 100 C overnight. Hörvath-Kawazoe model was used to estimate pore size distribution. IR spectra were obtained in a Nicolet 710 spectrometer (4 $\mathrm{cm}^{-1}$ resolution), using a conventional

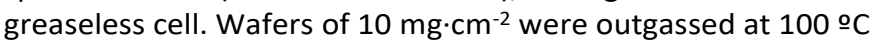
overnight. Solid state MAS-NMR spectra were recorded at room temperature in a Bruker AV-400 III HD spectrometer. ${ }^{27} \mathrm{Al}$ spectra were obtained using a $4 \mathrm{~mm}$ Bruker BL-4 probe. Pulses of $0.5 \mu$ s to flip the magnetization $\pi / 20 \mathrm{rad}$, and a recycle delay of $1 \mathrm{~s}$ were used. The ${ }^{1} \mathrm{H}$ to ${ }^{13} \mathrm{C}$ cross-polarization (CP) spectra were acquired by using a $90^{\circ}$ pulse for ${ }^{1} \mathrm{H}$ of $5 \mu \mathrm{s}$, a contact time of $2 \mathrm{~ms}$, and a recycle of $3 \mathrm{~s}$. The ${ }^{13} \mathrm{C}$ spectra were recorded with a $4 \mathrm{~mm}$ Bruker BL-4 probe and at a sample spinning rate of 10 $\mathrm{kHz}$. The ${ }^{1} \mathrm{H}$ to ${ }^{27} \mathrm{Al}$ cross-polarization $(\mathrm{CP})$ spectra were acquired with a bruker $3.2 \mathrm{~mm}$ probe using a rf-field for ${ }^{27} \mathrm{Al}$ of $8 \mathrm{kHz}$, spinning the sample at $16 \mathrm{kHz}$, a contact time of $1 \mathrm{~ms}$, and a recycle of $3 \mathrm{~s} .{ }^{13} \mathrm{C}$ and ${ }^{27} \mathrm{Al}$ were referred to adamantane and an aqueous solution of $\mathrm{Al}\left(\mathrm{NO}_{3}\right)_{3}$, respectively.

\section{Conflicts of interest}

There are no conflicts to declare.

\section{Acknowledgements}

The authors are grateful for financial support from the Spanish Government by MAT2014-52085-C2-1-P, MAT2017-82288-C21-P and Severo Ochoa Excellence Program SEV-2016-0683. J. M. $M$. thanks predoctoral fellowships from MINECO for economical support. The European Union is also acknowledged by ERCAdG-2014-671093-SynCatMatch.

\section{Notes and references}

1 B. Li, H.M. Wen, H. Wang, H. Wu, M. Tyagi, T. Yildirim, W. Zhou and B. Chen, J. Am. Chem. Soc., 2014, 136, 6207-6210.

2 R.B. Getman, Y.S. Bae, C. E. Wilmer and R.Q. Snurr, Chem. Rev., 2012, 112, 703-723.

3 M.P. Suh, H.J. Park,T.K. Prasad and D.W. Lim, Chem. Rev., 2012, 112, 782-835.

4 J. Liu, L. Chen, H. Cui, J. Zhang, L. Zhang and C.Y. Su, Chem. Soc. Rev., 2014, 43, 6011-6061.

5 A. H. Chughtai, N. Ahmad, H. A. Younus, A. Laypkov and F. Verpoort, Chem. Soc. Rev., 2015, 44, 6804-6849.

6 F.Y. Yi, D. Chen, M.K. Wu, L. Han and H-L. Jiang, Chem. Plus. Chem., 2016, 81, 675-690. 
7 R. B. Lin, S.Y. Liu, J.W. Ye , X.Y. Li and J.P. Zhang, Adv. Sci., 2016, 3, 1500434.

8 V. Stavila, A. Talin and M.D. Allendorf, Chem. Soc. Rev., 2014, 43, 5994-6010.

9 U. Díaz and A. Corma, Coord. Chem. Rev., 2016, 311, 85-124.

10 H. Li, M. Eddaoudi, M. O'Keeffe and O. M. Yaghi, Nature, 1999, 402, 276-279.

11 S. Inagaki, S. Guan, T. Ohsuna and O. Terasaki, Nature, 2002, 416 304-307.

12 S.Y. Ding and W. Wang, Chem. Soc. Rev., 2013, 42, 548-568.

13 A. Corma, H. García and F.X. Llabrés i Xamena, Chem. Rev., 2010, 110, 4606-4655.

14 C. Serre, F. Millange, C. Thouvenot, M. Nogués, G. Marsolier, D. Louër and G. Férey, J. Am. Chem. Soc., 2002, 124, 13519-13526.

15 C. N. R. Rao, H. S. S. Ramakrishna Matte and U. Maitra, Angew. Chem. Int. Ed., 2013, 52, 13162-13185.

16 G. Alberti, U. Costantinow, S. Allulli and N. Tomassini, J. Inorg. Nucl. Chem., 1978, 40, 1113-1117.

17 V. J. Margarit, M. E. Martínez-Armero, M. T. Navarro, C. Martínez and A. Corma, Angew. Chem. Int. Ed., 2015, 54, 13724-13728.

18 A. Corma, V. Fornés, S.B.C. Pergher, Th.L. Maesen and J.G. Buglass, Nature, 1998, 396, 353-356.

19 A. Corma, U. Díaz, M.E. Dómine and V. Fornés, J. Am. Chem. Soc., 2000, 122, 2804-2809.

20 C. González- Arellano, A. Corma, M. Iglesias and F. Sánchez, Adv. Synth. \& Catal., 2004, 346, 1758-1764.

21 M. Choi, K. Na, J. Kim, Y. Sakamoto, O. Terasaki and R. Ryoo, Nature, 2009, 461, 246-249.

22 M. Opanasenko, M. Shamzhy, F. Yu, W. Zhou, R. E. Morris and J. Cejka, Chem. Sci., 2016, 7, 3589-3601.

23 G. Bellussi, R. Millini, E. Montanari, A. Carati, C. Rizzo, W.O.N. Parker, G. Cruciani, A. De Angelis, L. Bonoldi and S. Zanardi, Chem. Commun., 2012, 48, 7356-7358.

24 G. Bellussi, A. Carati, E. Di Paola, R. Millini, W.O.N. Parker Jr, C. Rizzo and S. Zanardi, Microporous Mesoporous Mater., 2008, 113, 252-260.

25 G. Bellussi, A. Carati, C. Rizzo, U. Díaz Morales, S. Zanardi, W.O.N. Parker and R. Millini, World Patent, WO 2008/017513 A2, 2008.

26 G.E.Gomez, M.C. Bernini, E.V. Brusau, G.E. Narda, D. Vega, A.M. Kaczmarek, R. Van Deun and M. Nazzarro, Dalton Trans., 2015, 44, 3417-3429.

27 P. Amo-Ochoa, L. Welte, R. González-Prieto, P. J. Sanz Miguel, C. J. Gómez-García, E. Mateo-Martí, S. Delgado, J. Gómez-Herrero and F. Zamora, Chem. Commun., 2010, 46, 3262-3264.

28 T. Ródenas, I. Luz, G. Prieto, B. Seoane, H. Miro, A. Corma, F. Kapteijn, F. X. Llabrés i Xamena and J. Gascón, Nature Mater. 2015, 14, 48-55.

29 G. Cai and H-L. Jiang, Angew. Chem. Int. Ed., 2017, 56, 563-567.

30 S. J. Garibay and S. M. Cohen, Chem.Commun., 2010, 46, 77007702.

31 I. Senkovska, F. Hoffmann, M. Fröba, J. Getzschmann, W. Böhlmann and S. Kaskel. Microporous Mesoporous Mater., 2009, 122, 93-98.

32 C. G. Carson, K. Hardcastle, J. Schwartz, X. Liu, C. Hoffmann, R. A Gerhardt and R. Tannenbaum, Eur. J. Inorg. Chem., 2009, 16 2338-2343.

33 Q. Yang, S. Vaesen, M. Vishnuvarthan, F. Ragon, C. Serre, A. Vimont, M. Daturi, G. De Weireld and G. Maurin, J. Mater. Chem. 2012, 22, 10210-10220.

34 P. García-García, J. M. Moreno, U. Díaz, M. Bruix and A. Corma, Nat. Commun., 2016, 7, 10835.
35 J. M. Moreno, I. Navarro, U. Díaz, J. Primo and A. Corma, Angew. Chem. Int. Ed., 2016, 55, 11026-11030.

36 W. Ben-Cherif, R. Gharbi, H. Sebai, D. Dridi, N.A. Boughattas and M. Ben-Attia, C. R. Biol., 2010, 333, 214-219.

37 S.K. Ha, D. Shobha, E. Moon, M.A. Chari, K. Mukkanti, S.-H. Kim, K.-H. Ahn and S.Y. Kim, Bioorg. Med. Chem. Lett., 2010, 20, 39693971.

38 R. Varala, R. Enugala, S. Nuvula and S.R. Adapa, Synlett., 2006, 7, 1009-1011.

39 L-Z. Wang, X.Q. Li and Y.S. An, Org. Biomol. Chem., 2015, 13, 5497-5509.

40 Y. Huang, K. Khoury, T. Chanas and A. Dömling, Org. Lett., 2012, 14, 5916-5919.

41 J. J. Delpuech, M. R. Khaddar, A. A. Peguy and P. R. Rubini, J. Am Chem. Soc., 1975, 97, 3373-3379.

42 J. Gascón, A. Corma, F. Kapteijn and F.X. Llabres Xamena, ACS Catal., 2014, 4, 361-378.

43 P. García-García, M. Müller and A. Corma, Chem. Sci., 2014, 5, 2979-3007.

44 D. I. Jung, T. W. Choi, Y. Y. Kim, I. S. Kim, Y. M. Park, Y. G. Lee and D. H. Jung, Synth Commun, 1999, 29, 1941-1951.

45 M. Pozarentzi, J. Stephanidou-Stephanatou, C. A. Tsoleridis and A.Constantinos, Tetrahedron Lett., 2002, 43, $1755-1758$.

46 R. Varala, R. Enugala, S. R. Adapa and R. Srinivas, J. Braz. Chem. Soc., 2007, 18, $291-296$.

47 B. M. Reddy and P. M. Sreekanth, Tetrahedron Lett, 2003, 44, 4447-4449.

48 M. Tajbakhsh, M. M. Heravi, B. Mohajerani and A. N. Ahmadia, J Mol Catal A, 2006, 247, 213-215.

49 S. A. Majid, W. A. Khanday and R. Tomar, J. Biomed. Biotechnol., 2012, 1-6.

50 M. J. Climent, A. Corma, S. Iborra and L. L. Santos, Chem. Eur. J., 2009, 15, $8834-8841$.

51 M. A. Pasha and V. P. Jayashankara, Heterocycles, 2006, 68, 1017 1023.

52 M. S. Balakrishna and B. Kaboundin, Tetrahedron, 2001, 42, 1127-1129.

53 M. A. Chari and K. Syamasunder, Catal. Commun., 2005, 6, 67-70.

54 M. N. Timofeeva, S. A. Prikhodko, K. N. Makarova, M. E. Malyshev, V. N. Panchenko, A. B. Ayupov and S. H. Jhung, Reac. Kinet. Mech. Cat., 2017, 121, 689-699.

55 R. Fazaeli and H. Aliyan, Appl. Catal. A, 2007, 331, 78-83.

56 G. Huang, Q. Yang, Q. Xu, S-H. Yu and H-L. Jiang, Angew. Chem. Int. Ed. 2016, 55, 7379-7383.

57 M. Jeganathan and K. Pitchumani, ACS Sustainable Chem. Eng., 2014, 2, 1169-1176. 